\title{
The Spatial Order of Horizontal Cells Is Not Affected by Massive Alterations in the Organization of Other Retinal Cells
}

\author{
Chiara Rossi, Enrica Strettoi, and Lucia Galli-Resta \\ Istituto di Neuroscienze del Consiglio Nazionale delle Ricerche, Sezione di Neurofisiologia, 56100 Pisa, Italy
}

To test whether retinal mosaics develop through interactions that are restricted primarily to the mosaic cells, we studied the horizontal cell mosaic in mutant mice $(r d / r d$ and $r d / b c l 2)$ displaying severe retinal abnormalities. These mutants show that the horizontal cell mosaic develops normally even if these cells lack part of their synaptic input, have anomalous morphologies, eventually decrease in number, and reside in an abnormally packed retinal layer. These data strongly support a developmental design in which the final position of each cell in a retinal mosaic is controlled by interactions between homotypic cells and is independent of other cell types.

The present analysis is also an investigation of the effects of photoreceptor degeneration on the horizontal cells in an established animal model of retinitis pigmentosa, the $r d / r d$ mouse. We find that the organization of the horizontal cell mosaic resists photoreceptor degeneration and, furthermore, that bcl2 overexpression prevents the partial loss of horizontal cells secondary to photoreceptor loss. Secondary degeneration hampers attempts to restore retinal function by transplanting photoreceptors or promoting their survival. The anti-apoptotic gene bcl2 appears to be a promising tool to rescue inner retinal neurons, increasing the probability that photoreceptor rescue or substitution may be beneficial to subjects suffering from retinal degenerative diseases.

Key words: retina; retinitis pigmentosa; horizontal cells; mosaic; antibody; development

\section{Introduction}

The retina consists of microcircuits arranged in a sheet. These must sample every part of the visual scene. Facing these demands, cells of the same type are commonly regularly spaced, forming what have become known as retinal mosaics. Pioneering studies have shown the orderly spatial arrangement of cone photoreceptors, horizontal cells, and ganglion cells (Wässle and Riemann, 1978). Nonrandom spacing has now been confirmed for most retinal cell types (Wässle and Boycott, 1991; Cook and Chalupa, 2000).

Mosaics arise early in development, and recent studies show that minimal spacing rules between like cells are sufficient to form most mosaics, so that each mosaic could develop independently of other neuronal types in the retina (Cook and Chalupa, 2000; Galli-Resta, 2002). Experimental testing of this developmental rule, however, has only just begun (Galli-Resta et al., 1999; Galli-Resta, 2000; Rockhill et al., 2000; Raven and Reese, 2003).

As a contribution to this analysis, we focused here on the horizontal cell mosaic in animal models of retinal degenerative disease, in which we can simultaneously investigate how pathology affects mosaic organization and whether retinal mosaics de-

Received May 22, 2003; revised Sept. 2, 2003; accepted Sept. 9, 2003.

This work was supported by National Institutes of Health Grant R01 EY12654 and by TeleThon Project E.0833 to E.S. and TeleThon Project E.1333 to L.G.R.

Correspondence should be addressed to Dr. Enrica Strettoi, Istituto di Neuroscienze del Consiglio Nazionale delle Ricerche, Sezione di Neurofisiologia, via Giuseppe Moruzzi 1, 56100 Pisa Italy. E-mail: enrica.strettoi@in.cnr.it. Copyright $\odot 2003$ Society for Neuroscience $\quad$ 0270-6474/03/239924-05\$15.00/0 velop independently of abnormalities in other neuronal types. We analyzed here $r d / r d$ and $r d / b c l 2$ mutants, in which the horizontal cells lack normal synaptic input, have anomalous morphologies, eventually decrease in number, and reside in an abnormally packed retinal layer.

In the $r d / r d$ mouse, photoreceptors undergo a progressive fast degeneration, which is almost complete by 1 month of age (Carter-Dawson et al., 1978; Jimenez et al., 1996). Transient synaptic contacts between rods and horizontal cells develop to an initial stage, but only some of them mature (Blanks et al., 1974). Severe alterations in the morphology of horizontal cells are observed in the $r d / r d$ mutant, comprising hypertrophy and loss of processes. By 3 months, horizontal cells also decrease in number (Strettoi and Pignatelli, 2000). This mutant allowed us to test whether horizontal cells form a mosaic independently of the partial loss of synaptic input and notwithstanding their morphological abnormalities. We also tested the effects of partial cell loss on mosaic organization.

We generated the $r d / \mathrm{bcl} 2$ mutant by crossing the $r d / r d$ mouse with a transgenic overexpressing the anti-apoptotic gene bcl2 in all CNS neurons but the photoreceptors. As a consequence of a reduced developmental cell death, most neuronal types are supernumerary in this mutant, including most cells in the retinal inner nuclear layer (INL) but not the horizontal cells. This mutant allowed us to test whether the horizontal cell mosaic is normal despite abnormal development of synaptic input, eventual loss of input cells, and an increased number of cells in the very layer in which the horizontal cells reside. We also tested the pro- 
tective effects of bcl2 overexpression on the secondary loss of horizontal cells induced by the $r d$ mutation.

\section{Materials and Methods}

Animal handling, tissue acquisition, and histological treatment. C57BL/6 J mice (Nossan, Milan, Italy) [from now on referred to as wild type (wt)], $\mathrm{C} 3 \mathrm{H} r d / r d$ mice (Iffa Credo, Reims, France), and bcl2 overexpressing transgenic mice on a C57BL background (a strain originated by J.-C. Martinou, University of Geneva, Switzerland) were bred in the laboratory colony. All procedures using mice were conducted in accordance with national regulations and the Association for Research in Vision and Ophthalmology statement. Whole-mount retinas were analyzed in this study; three retinas for each strain were taken at 1 and 3.5 months of age. Animals were anesthetized with Avertin $(0.1 \mathrm{ml} / 5 \mathrm{gm}$ body weight, i.p.), perfused with $4 \%$ paraformaldehyde in $0.1 \mathrm{~m}$ phosphate buffer for sections, or the eyes were enucleated and fixed for $30 \mathrm{~min}$ for whole mounts. Horizontal cells were labeled using a calbindin D 28K monoclonal antibody [(Sigma, St. Louis, MO; clone CB 955 1:100 (sections) or 1:1000 (whole mounts)], followed by Oregon-Green-488 secondary antibody (1:200; Molecular Probes, Eugene, OR). Nuclei were stained with redPOPO3 (Molecular Probes). Dissection, sectioning, and immunostaining are described in Strettoi and Pignatelli (2000).

Generation and characterization of $\mathrm{rd}^{+/+}, b c l 2^{+/-}$mutants. To obtain $r d^{+/+}, \mathrm{bcl} 2^{+/-}$mice, $r d^{+/+}$females were bred with bcl2-overexpressing transgenic males. Bcl2 females have a closed vagina and cannot be mated. $\mathrm{Bcl} 2$ transgenics are therefore heterozygous. The offspring (F1 generation), heterozygous for the $r d$ mutation, were analyzed by PCR to identify the animals carrying the human bcl 2 transgene; genomic DNA was extracted from tail biopsies, using the primers 5' -ATGAGCCTTGGGACTGTGAA-3' and 5'-GAAGACTCTGCTCAGTTTGG-3'

F1 males $r d^{+/-}$, bcl2 ${ }^{+/-}$were backcrossed with females $r d^{+/+}$. In F2 generation, the males carrying the homozygous $r d$ mutation and the human bcl2 transgene were identified by PCR analysis [for bcl2 primers see above; for $r d$ primers see Gustincich et al. (1997)]. These founders were always backcrossed with $r d^{+/+}$females, and the offspring were tested by PCR for bcl 2 primers. The presence of the human $\mathrm{Bcl} 2$ protein in horizontal cells of $r d / b c l 2$ mice was confirmed by double-labeling immunocytochemistry using calbindin and anti-human Bcl2-fluorescein antibodies (Dako, Carpinteria, CA; clone 124). The latter does not crossreact with endogenous mouse Bcl2.

Data sampling. Samples were acquired using a Leica (Nussloch, Germany) TCS-NT confocal microscope. In each whole-mount retina, 20 $\left(250 \times 250 \mu \mathrm{m}^{2}\right)$ regularly spaced fields were sampled along the four major retinal axes. Retinal areas and the coordinates of cell positions in each sample were obtained using an Image Analyzer (Imaging, Ontario, Canada). The total number of horizontal cells in each retina was determined multiplying the retinal area by the average density of horizontal cells in that retina.

Data analysis, statistical analysis, and simulations. To study the organization of the horizontal cell mosaic, we analyzed three distributions: the nearest neighbor distribution, by which we obtained the regularity index, defined as the ratio between the mean and the SD of the distribution of nearest-neighbor distances; the distribution of the Delaunay segments (segments that link each cell with its surrounding homotypic neighbors); and the distribution of the Delaunay areas (enclosed by the Delaunay segments). To determine the Delaunay triangulation, a domain is defined for each cell containing all the points in the plane that are closer to that cell than to any other cell in the mosaic (Voronoi domain). Delaunay segments link cells with adjacent domains. We have chosen the Delaunay triangulation because it provides very reliable statistics: each cell has one nearest neighbor, one Voronoi domain, and an average of 2.5 Delaunay segments.

Although the number of horizontal cells is constant, adult mouse retinas vary in areas, with a consequent variability in horizontal cell density (Jeon et al., 1998). This variability in cell density obviously affects cell spacing, but we found that the Delaunay curves obtained for different wt animals perfectly superimpose with one another if we first normalize the average density of horizontal cells in each retina to the average wt density.

In other words, the wt retinas have a constant number of horizontal cells and a common geometry of horizontal cell distribution, so that larger retinas space them proportionately more than smaller retinas. Therefore, to analyze true differences in the geometry of the horizontal cell mosaics between wt and mutant mice, we adopted this normalization procedure whenever the number of cells in the retina analyzed were within the normal wt range. This normalization was not performed in the cases of the 3.5-month-old $r d$ mutants that have a decreased number of horizontal cells (Strettoi and Pignatelli, 2000). Plots and frequency histograms were produced by means of Origin 5.0 (Microcal). Statistical comparisons between histograms from wt and mutant animals were performed using the bootstrap analysis. This analysis avoids assumptions about the data distribution and takes into account the variability within each dataset. This is possible because the bootstrap uses computational power to obtain a numerical estimate of the quantity of interest, in this case the difference between the distributions associated with the mosaics of the wt and the mutant retinas. Briefly, from a data set $x=\left(x_{1}, x_{2}, \ldots\right.$ $x_{\mathrm{N}}$ ) of size $N$, a sample of the same size is obtained by drawing with replacement elements from $x$. The bootstrap sample is denoted $x^{*}=\left(x_{1}^{*}\right.$, $x_{2}^{*}, \ldots, x_{\mathrm{N}}{ }^{*}$ ). Each of the $x_{j}^{*}$ is one of the original $x$ value, randomly selected. For example $x_{1}{ }^{\star}=x_{2}, x_{2}{ }^{\star}=x_{5}, x_{3}{ }^{\star}=x_{5}$. The bootstrap estimate of the quantity of interest is obtained as follows: (1) a large number $(B)$ of independent bootstrap samples is drawn from the data, (2) the quantity of interest is calculated for each bootstrap sample, and (3) mean and SD of quantity of interest are derived from the $B$ bootstrap samples. In our case a bootstrap sample is obtained by randomly drawing (with replacement) $N$ wt histograms and $M$ mutant histograms, where $N$ is the number of wt histograms and $M$ the number of mutant histograms obtained. For each bootstrap sample we computed the total wt histogram and the total histogram for the mutant data, normalized them, and subtracted one from the other to obtain the difference. From the 10,000 bootstrap samples we derived the mean and SD for the count contained in each bin of the different histograms. This was performed for every comparison shown in Figures 3 and 4, enabling us to determine the extent to which the wt and mutant histograms differed. A difference between wt and mutant is scored as significant when the bootstrap estimate of the quantity of interest remains for all bins within the difference obtained comparing independent data sets from wt animals. The wt samples showed a variability $\leq 13 \%$.

Bootstrap was performed by means of a custom-made program (GalliResta et al., 1999). A detailed explanation of the bootstrap method is in Efron and Tibshirani (1991).

The random elimination of horizontal cells in the wt samples was performed with a custom-made program.

\section{Results}

Significant abnormalities of retinal architecture are evident in the $r d / r d$ and $r d / b c l 2$ mice even in low-power vertical sections (Fig. $1 A-C)$. At 1 month of age, both mutants lack photoreceptors. In addition, the $r d / b c l 2$ displays an abnormally thick INL in which many more cells than normal are packed (Fig. 1C), as in the parent bcl2 strain (Strettoi and Volpini, 2002). The horizontal cells (green) are in a monolayer in both mutants, appropriately positioned in the outer part of the INL. Examples of the horizontal cell organization as seen in whole-mount retinas of the wt and mutant strains are shown in Figure $1 D-F$. The number of horizontal cells in either mutant is not significantly different from that observed in the wt animals at 1 month of age, as shown in Figure $2 \mathrm{~A}$. At 3.5 month of age, the $r d / r d$ mutants show a slight decrease in the number of horizontal cells (Fig. 2A, left), as reported previously (Strettoi and Pignatelli, 2000).

To test the organization of the horizontal cell mosaic we first analyzed the regularity index, a common measure of regularity, defined as the ratio between the mean and the SD of the distribution of nearest neighbor distances. As shown in Figure $2 B$, this measure did not provide significantly different values for the wt and the two strains of mutant mice ( $t$ test significance level, $p=$ 0.01 ). To further study the spatial organization of horizontal 
cells, we analyzed the distribution of Delaunay segments, which link each horizontal cell to its surrounding homotypic neighbors, and the distribution of the areas of the triangles enclosed by the Delaunay segments (Fig. 3A). This analysis allows us to measure the regularity of cell spacing within the mosaics and that of the tiling associated with the mosaic. The corresponding histograms (Fig. $3 B-E$ ) show that at 1 month of age, there is no difference between the geometrical organization of the horizontal cell mosaic in the wt and the $r d / r d$ or $r d /$ bcl 2 mutants (bootstrap analysis).

At 3.5 months of age a difference in the horizontal cell mosaic becomes apparent between the wt and the $r d / r d$ mutant in the Delaunay triangulation (Fig. $4 A$ ), whereas there is no difference between wt and $r d /$ bcl2 (Fig. 4B) (bootstrap analysis). At this age, however, the $r d / r d$ mutants have lost some horizontal cells (Fig. 2A) (Strettoi and Pignatelli, 2000), suggesting that a difference in their mosaic could simply be the result of cellular loss. To test this hypothesis, we produced simulations by taking the wt samples of the horizontal cell mosaic and randomly eliminating cells until their number had dropped to the $r d / r d$ level (Fig. 4C). These simulations have Delaunay segment distributions indistinguishable from the $r d / r d$ case (Fig. $4 D$ ), suggesting that the cell loss by itself can explain the observed change in the horizontal cell mosaic at this age.

\section{Discussion}

We have found that the spatial organization of the horizontal cells is not affected in mutant retinas displaying profound alterations in the horizontal cell synaptic input and in the density of the cell layer where the horizontal cells are located. This analysis focused on two mutant strains, the $r d / r d$ and $r d / \mathrm{bcl} 2$ mice. In the $r d / r d$ mouse the horizontal cells lack functional input from rod photoreceptors. In this mutant, the rapid and massive degeneration of the rod photoreceptors is complete within the first weeks of life, paralleled by a slower loss of cones (LaVail et al., 1997). Rod bipolar cells are never observed to form appropriate synaptic contacts with photoreceptors (Blanks et al., 1974; Strettoi and Pignatelli, 2000). This mutant thus allows testing of the dependence of the horizontal cell mosaic on the cell providing the major synaptic input.

In the $r d / b l c 2$ mutant the horizontal cells are in normal number, but lack functional input as in the $r d$ mutation, and reside in an abnormally densely populated layer, because the number of cells forming the INL is significantly higher than in the wt animals (Fig. 1C), as in the bcl2 parent strain (Strettoi and Volpini, 2002). This mutant allows testing of the independence of the horizontal cell mosaic from the density of cells in the layer where they reside and in general from the cell populations in the INL.

We found that in both mutants the organization of the horizontal cell mosaic is indistinguishable from normal. The conclusion seems inescapable that the maintenance of the regular spacing of horizontal cells does not require normal synaptic interactions or normal cell packing.
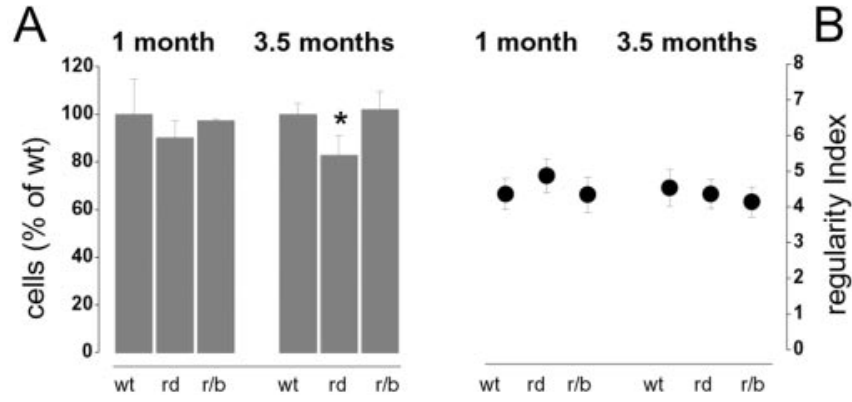

Figure 2. A, The number of horizontal cells is normal in the $r d / r d$ and $r d / b c \mid 2$ mice at 1 month of age. It becomes slightly but significantly lower in the $r d / r d$ mutant by 3.5 month of age. Such a decrement is not observed in the $\mathrm{rd} / \mathrm{bcl} 2$ mutants. $B$, No significant difference is observed in the regularity index between the wt and either mutants. The regularity index is the ratio between the mean and SD of the distribution of nearest-neighbor distances.

These findings indeed show that the horizontal cell mosaic can form even when there are profound alterations in other cell types at the time the mosaic develops. In the $r d / r d$ mouse the horizontal cell mosaic attains a mature spatial organization not earlier than postnatal day $(\mathrm{P})$ 10, when horizontal cells become positioned in a single monolayer (Schnitzer and Rusoff, 1984; Reese et al., 1999). At this time both mutants that we analyzed already display severe retinal abnormalities. At P10 the $r d / r d$ mice have abnormal and reduced synaptic contacts between photoreceptors and horizontal cells and already display conspicuous photoreceptor degeneration (Blanks et al., 1974). These alterations affect the morphology of the horizontal cells [which show a 10-fold increase in axonal sprouting by the second postnatal week (Strettoi et al., 2002)] but not their spatial ordering.

In the $r d / b c l 2$ mutants the horizontal cells are confronted with the photoreceptor loss derived from the parent $r d$ mutation; they 


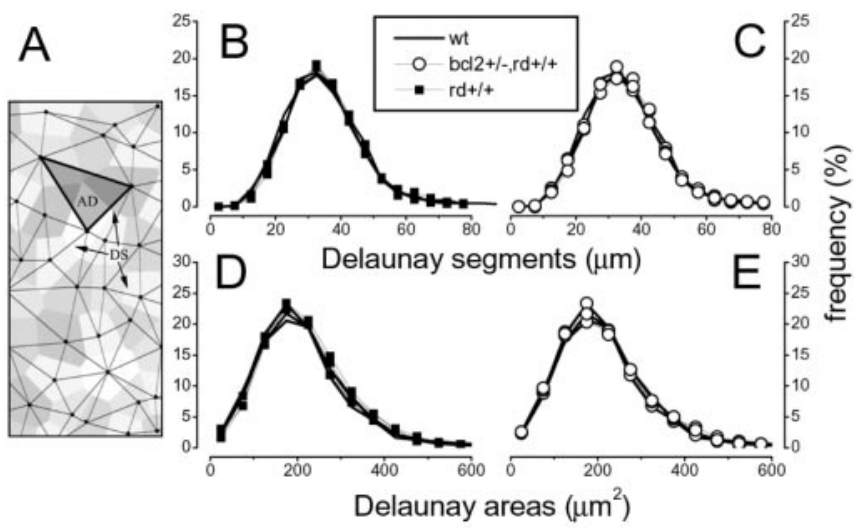

Figure 3. No significant difference is observed between the Delaunay triangulation associated with the horizontal cell mosaic in the wt or in either mutation at 1 month of age. $A$, An example of the Delaunay triangulation. Delaunay segments link each cell with its surrounding neighbors. $B, C$, No significant difference is observed in the distribution of Delaunay segments between the $r d / r d$ and the wt $(B)$ or the wt and the $r d / b c 12$ mutant ( $C$. D, E, The distribution of Delaunay areas is not statistically different between the wt and either mutant. (KolmogorovSmirnov test between average distribution; in each case $p<0.05$. Differences are also not statistically significant when analyzed with the bootstrap method.)

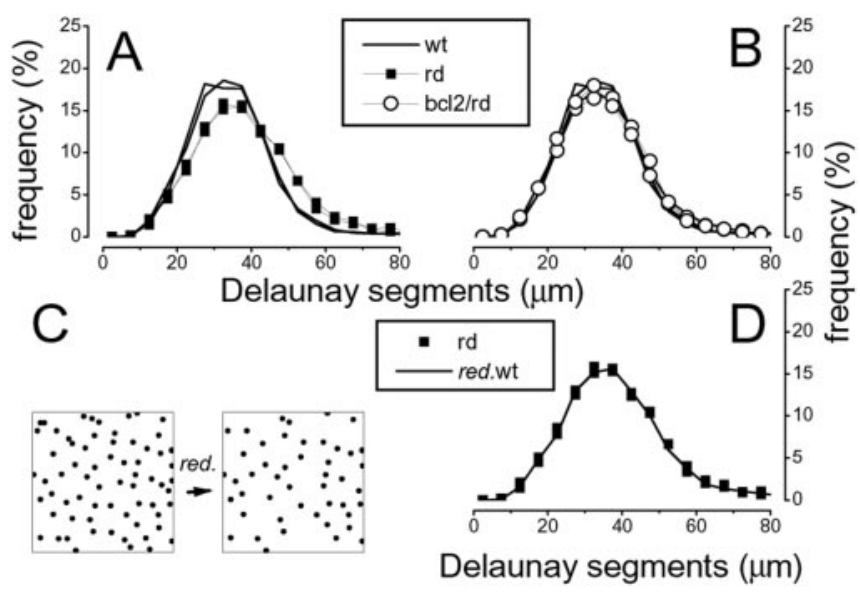

Figure 4. Quantitative analysis of the horizontal cell mosaic in the $\mathrm{wt}, r d / r d$, and $r d / \mathrm{bcl} 2$ mutations at 3.5 months of age. $A, B$, A slight but significant difference is observed between the distribution of Delaunay segments of the $r d / r d$ and wt $(A)$, whereas the $r d / b c 12$ remains indistinguishable from the wt $(B) . C, D, B y 3.5$ months of age, the $r d / r d$ has lost a small but significant number of cells (Fig. 2A). The random elimination of cells in the wt samples until these had the same number of cells found in the $r d / r d$ ( $C$ suffices to reproduce the $r d / r d$ distribution of Delaunay segments $(D)$.

also reside in an abnormally dense layer, for the increased cell survival caused by bcl2 overexpression (Strettoi and Volpini, 2002). Still, we found that the spatial organization of the horizontal cells is normal.

These data nicely complement previous findings showing that the horizontal mosaic forms independently of the developmental abnormalities brought about by albinism or of the presence of cone photoreceptors (Raven and Reese, 2002, 2003). Taken together the results of this and previous studies (Raven and Reese, 2002 , 2003) strongly suggest that the spatial organization of the horizontal cell mosaic is largely independent of other cells types.

The hypothesis that most retinal mosaics develop independently of each other originates from studies showing that mosaics formed by cells of different types lack any spatial correlation (Rockhill et al., 2000) and from the observation that in most retinal mosaics the only detectable pattern is a minimal spacing between mosaic cells (Galli-Resta, 1998, 2000). This minimal spacing rule is sufficient to simulate the geometry of mosaics, suggesting that interactions between the mosaic cells suffice for mosaic formation (Galli-Resta et al., 1997; Galli-Resta, 1998, 2001). The minimal spacing rule must be complemented by additional constraints when a single cell population splits into two mosaics [the ON-OFF mosaics of ganglion cells (Bodnarenko and Chalupa, 1993; Zhan and Troy, 2000) or in the case of spatially correlated mosaics (Kouyama and Marshak, 1997)].

The independent assembly of most retinal mosaics is an important feature of retinal development. In a system in which 50 or so cell types are generated (Masland, 2001), it is not difficult to envision the need for spatial positioning to be controlled independently of the events by which the cells are originally specified (Rockhill et al., 2000). The different cell types appear to be specified by a combination of a progressive restriction in the potential of the cell progenitors and inductive events (Perron and Harris, 2000; Livesey and Cepko, 2001). If the spatial positioning of cells could not undergo additional and separate controls it would be difficult to explain how each cell type would regularly tile the retina and yet be positioned independently of other cells types.

A much more effective developmental design, consistent with the experimental observations so far available, is that cell genesis and cell positioning are determined separately. Once generated, cells migrate to their layer, and a simple rule controlling the spacing between cells of the same type suffices to control mosaic formation (Galli-Resta et al., 1997; Galli-Resta, 2001). This ensures uniform coverage of the retina by each cell type, independent of the mechanisms that control the genesis of the different retinal cells.

The finding that the horizontal cell mosaic is unaffected in the $r d / r d$ and $r d / b c l 2$ mice is also relevant in the context of retinal degeneration, because the $r d / r d$ mutation is a classic animal model for retinitis pigmentosa (Chang et al., 2002). Promising attempts are in progress to prevent photoreceptor loss or restore functional photoreceptors in this family of diseases (Frasson et al., 1999; Acland et al., 2001; Lund et al., 2001). The degree of preservation of the inner retina is critical for the potential of these attempts to lead to functional recovery. Although recent studies show significant alterations in the horizontal cell morphology (Strettoi and Pignatelli, 2000; Strettoi et al., 2002), this study reveals that the horizontal cells retain the even coverage of the retina that ensures the physiological tiling of the visual scene.

Finally, this study provides new insight into the mechanisms controlling horizontal cell death, as revealed by the $\mathrm{rd} / \mathrm{bcl} 2 \mathrm{mice}$, in which bcl 2 overexpression is capable of preventing the secondary loss of horizontal cells observed in the central retinas of $r d / r d$ mice at 3.5 month of age. Thus, this anti-apoptotic agent could be used to rescue inner retinal neurons from secondary degeneration, enlarging the time window in which receptor transplants or substitution may be beneficial to subjects suffering from retinal degenerative diseases.

\section{References}

Acland GM, Aguirre GD, Ray J, Zhang Q, Aleman TS, Cideciyan AV, PearceKelling SE, Anand V, Zeng Y, Maguire AM, Jacobson SG, Hauswirth WW, Bennett J (2001) Gene therapy restores vision in a canine model of childhood blindness. Nat Genet 28:92-95.

Blanks JC, Adinolfi AM, Lolley RN (1974) Photoreceptor degeneration and synaptogenesis in retinal-degenerative (rd) mice. J Comp Neurol 156:95-106.

Bodnarenko SR, Chalupa LM (1993) Stratification of ON and OFF ganglion cell dendrites depends on glutamate-mediated afferent activity in the developing retina. Nature 364:144-146. 
Carter-Dawson LD, LaVail MM, Sidman RL (1978) Differential effect of the rd mutation on rods and cones in the mouse retina. Invest Ophthalmol Vis Sci 17:489-498.

Chang B, Hawes NL, Hurd RE, Davisson MT, Nusinowitz S, Heckenlively JR (2002) Retinal degeneration mutants in the mouse. Vision Res 42:517-525

Cook JE, Chalupa LM (2000) Retinal mosaics: new insights into an old concept. Trends Neurosci 23:26-34.

Efron B, Tibshirani RJ (1991) Statistical data analysis in the computer age. Science 253:390-395.

Frasson M, Sahel JA, Fabre M, Simonutti M, Dreyfus H, Picaud S (1999) Retinitis pigmentosa: rod photoreceptor rescue by a calcium-channel blocker in the rd mouse. Nat Med 5:1183-1187.

Galli-Resta L (1998) Patterning the vertebrate retina: the early assembly of retinal mosaics. Semin Cell Dev Biol 9:279-284.

Galli-Resta L (2000) Local, possibly contact-mediated signaling restricted to homotypic neurons controls the regular spacing of cells within the cholinergic arrays in the developing rodent retina. Development 127:1499-1508.

Galli-Resta L (2001) Assembling the vertebrate retina: global patterning from short-range cellular interactions. NeuroReport 12:A103-A106.

Galli-Resta L (2002) Putting neurons in the right places: local interactions in the genesis of retinal architecture. Trends Neurosci 25:638-643.

Galli-Resta L, Resta G, Tan S-S, Reese B (1997) Mosaics of Islet-1-expressing amacrine cells assembled by short-range cellular interactions. J Neurosci 17:7831-7838.

Galli-Resta L, Novelli E, Kryger Z, Jacobs G, Reese B (1999) Modeling the mosaic organization of rod and cone photoreceptors with a minimal spacing rule. Eur J Neurosci 11:1438-1446.

Gustincich S, Feigenspan A, Wu DK, Koopman LJ, Raviola E (1997) Control of dopamine release in the retina: a transgenic approach to neural networks. Neuron 18:723-736.

Jeon C-J, Strettoi E, Masland R (1998) The major cell populations of the mouse retina. J Neurosci 18:8936-8946.

Jimenez AJ, Garcia-Fernandez JM, Gonzalez B, Foster RG (1996) The spatio-temporal pattern of photoreceptor degeneration in the aged $\mathrm{rd} / \mathrm{rd}$ mouse retina. Cell Tissue Res 284:193-202.

Kouyama N, Marshak DW (1997) The topographical relationship between two neuronal mosaics in the short wavelength-sensitive system of the primate retina. Vis Neurosci 14:159-167.

LaVail MM, Matthes MT, Yasumura D, Steinberg RH (1997) Variability in rate of cone degeneration in the retinal degeneration (rd/rd) mouse. Exp Eye Res 65:45-50.

Livesey FJ, Cepko CL (2001) Vertebrate neural cell-fate determination: lessons from the retina. Nat Neurosci 2:109-118.

Lund RD, Adamson P, Sauve Y, Keegan DJ, Girman SV, Wang S, Winton H Kanuga N, Kwan AS, Beauchene L, Zerbib A, Hetherington L, Couraud PO, Coffey P, Greenwood J (2001) Subretinal transplantation of genetically modified human cell lines attenuates loss of visual function in dystrophic rats. Proc Natl Acad Sci USA 98:9942-9947.

Masland RH (2001) Neuronal diversity in the retina. Curr Opin Neurobiol 11:431-436.

Perron M, Harris WA (2000) Determination of vertebrate retinal progenitor cell fate by the Notch pathway and basic helix-loop-helix transcription factors. Cell Mol Life Sci 57:215-223.

Raven MA, Reese BE (2002) Horizontal cell density and mosaic regularity in pigmented and albino mouse retina. J Comp Neurol 454:168-176.

Raven MA, Reese BE (2003) Mosaic regularity of horizontal cells in the mouse retina is independent of cone photoreceptor innervation. Invest Ophthalmol Vis Sci 44:965-973.

Reese B, Necessary B, Tam P, Faulkner-Jones B, Tan S (1999) Clonal expansion and cell dispersion in the developing mouse retina. Eur J Neurosci 11:2965-2978.

Rockhill RL, Euler T, Masland RH (2000) Spatial order within but not between types of retinal neurons. Proc Natl Acad Sci USA 97:2303-2307.

Schnitzer J, Rusoff A (1984) Horizontal cells of the mouse retina contain glutamic acid decarboxylase-like immunoreactivity during early developmental stages. J Neurosci 4:2948-2955.

Strettoi E, Pignatelli V (2000) Modifications of retinal neurons in a mouse model of retinitis pigmentosa. Proc Natl Acad Sci USA 97:11020-11025.

Strettoi E, Volpini M (2002) Retinal organization in the bcl-2overexpressing transgenic mouse. J Comp Neurol 446:1-10.

Strettoi E, Porciatti V, Falsini B, Pignatelli V, Rossi C (2002) Morphological and functional abnormalities in the inner retina of the $\mathrm{rd} / \mathrm{rd}$ mouse. J Neurosci 22:5492-5504.

Wässle H, Boycott BB (1991) Functional architecture of the mammalian retina. Physiol Rev 71:447-480.

Wässle H, Riemann HJ (1978) The mosaic of nerve cells in the mammalian retina. Proc R Soc Lond B Biol Sci 200:441-461.

Zhan XJ, Troy JB (2000) Modeling cat retinal beta-cell arrays. Vis Neurosci $17: 23-39$. 